\title{
Magnetic Separation of Graphite and Magnesia
}

\author{
C. UYedA* AND K. Hisatoshi \\ Institute of Earth \& Space Science, Graduate school of Science Osaka University Toyonaka Osaka 560-0043 Japan
}

\begin{abstract}
An ensemble of graphite and magnesia particles was successfully separated into different groups of materials by translating them in an area of magnetic field produced by a $\mathrm{NdFeB}$ magnetic-circuit. The absolute value of diamagnetic susceptibility $\left|\chi_{\text {dia }}\right|$ was $2.6 \times 10^{-7} \mathrm{emu} / \mathrm{g}$ in magnesia; previously the separation of diamagnetic grains was possible in materials with $\left|\chi_{\text {dia }}\right|$ values above $5 \times 10^{-7} \mathrm{emu} / \mathrm{g}$. Terminal velocity observed in a area outside the magnetic field in the magnesia particles having different mass between 0.12 to $0.35 \mathrm{mg}$, converged to a constant value $1.27 \pm 0.05 \mathrm{~cm} / \mathrm{s}$; the value was consistent with a theoretical value based on a simple conversion rule between magnetic potential and kinetic energy assumed in the particles. Similar result was obtained for the graphite particles. The technical improvements introduced in the present study to translate the $\mathrm{MgO}$ particles can be applied to realize magnetic separation of diamagnetic materials in general.
\end{abstract}

DOI: 10.12693/APhysPolA.133.694

PACS/topics: $75.90 .+\mathrm{w}$

\section{Introduction}

In a recent report, a mixed ensemble of weak magnetic (i.e. diamagnetic \& paramagnetic) particles was dynamically separated into fractions of different materials by applying a low field produced by $\mathrm{Nd}$ magnetic circuit (maximum field $<0.8 \mathrm{~T}$ ) [1]. Formerly, dynamical motions of weak magnetic materials were not expected to occur unless an ultra-strong field above $10 \mathrm{~T}$ was introduced in the experiment [2]. In order to realize the above separation, the particles were translated in a area of monotonically decreasing field; they were allowed to translate freely in a diffused area $(\sim 100 \mathrm{~Pa})$ by applying a short microgravity condition. The sample pieces consisted of diamagnetic bismuth, diamond \& graphite as well as two paramagnetic olivine with different Fe concentrations. Furthermore, material of the separated particles could be identified from its magnetic susceptibility $\chi$ (per unit mass) that was determined from the translating velocity. A simple method is required in analytical science to extract and identify minor solid particles from a ensemble of heterogeneous particles, and the magnetic separation can be used in such method of pretreatment [1].

In the present report, improvements were made in the procedure to realize the separation of diamagnetic materials that posses small $\left|\chi_{\text {dia }}\right|$ value [3]. The mass dependence of the field-induced translation were examined in graphite and magnesia particles to examine the efficiency of energy conversion rule during the process of magnetic separation.

\section{Experimental}

The short microgravity $(\mu g)$ condition used in the experiment was produced by a drop shaft that had a length of $1.8 \mathrm{~m}$. The experimental setup to observe the grain

*corresponding author; e-mail: uyeda@ess.sci.osaka-u.ac.jp motion was installed in a wooden drop box $(30 \times 3 \times 20$ $[\mathrm{cm}])$ which was attached to the ceiling of the drop shaft using an electromagnet; free fall of the drop box started by shutting down the power supply of the electromagnet [1]. The effective microgravity duration of the system was about 0.5 seconds. The setup installed in the drop box consisted of a small magnetic circuit (maximum field intensity: $0.86 \mathrm{~T}$ ), a transparent vacuum chamber that enclosed the magnetic circuit and a high speed camera with an illumination system to observe the translation.

The magnetic circuit that produced a suitable field gradient to induce the grain translation was composed of a pair of $\mathrm{NeFeB}$ plates $(4.0 \times 3.0 \times 1.0[\mathrm{~cm}])$ as shown in the upper part of Fig. 1. A direction which had the largest gradient of monotone decreasing field was defined as a $+x$-axis, whereas the $+z$-axis was defined along the magnetic line of force produced between the $\mathrm{N}$ pole and $\mathrm{S}$ pole in the circuit. A collecting plate was placed in the translating areas to examine the result of magnetic separation. The grain ensemble was set at a position located along the $x$-axis; this position was at the edge of the magnetic circuit (see Fig. 1) with field intensity $B_{0}$. The grain ensemble consisted of sub-millimeter size pieces of graphite and magnesia; they were produced from highquality bulk materials. Details of chemical and magnetic analysis of the materials have been described elsewhere [4]. Mass $m$ of individual particles is listed in Table I. The circuit together with the particles was enclosed in the glass tube, and the inner pressure was reduced to $\sim 100 \mathrm{~Pa}$ to eliminate the effect of air resistance.

Shortly after the beginning of $\mu g$ condition, the sample stage set inside the stage-holder (attached at the above position) was levitated for about $0.5 \mathrm{~mm}$; this movement was effective in releasing the samples in the diffuse $\mu g$ area with small initial momentum; the stage was spontaneously levitated by a small field gradient located in the $+\mathrm{z}$ direction [1]. In a microgravity experiment, it was generally difficult to release a substance in a diffuse $\mu g$ area with small initial velocity. 


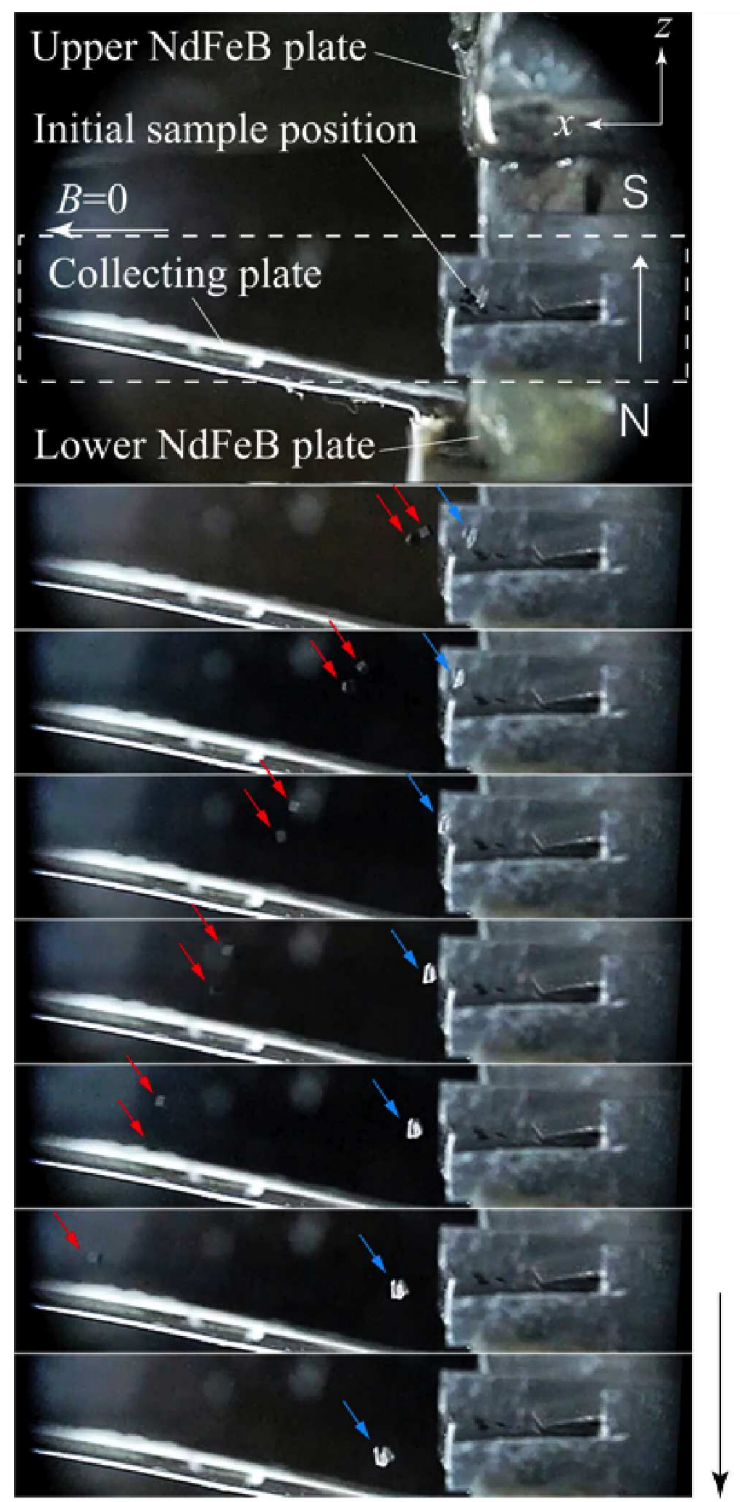

Time interval $0.05 \mathrm{~s}$

\section{Red arrow:graphite \\ Blue arrow: $\mathrm{MgO}$}

Fig. 1. Time sequence photograph of magnetic separation observed between graphite and magnesia particles that was observed in a short microgravity condition.

In order to minimize the effect of Coulomb attraction force which prevented the release of grains, deionization of sample particle was performed in the present study for a long duration $(\sim 1 \mathrm{hr})$ using a commercial apparatus before it was set on the sample stage. After setting the particles on the stage, surface charge of the particles together with the stage was again neutralized by a portable deionizer. Local field distribution around the sample holder was altered as well by attaching additional magnetic plates near the holder, which increased the field

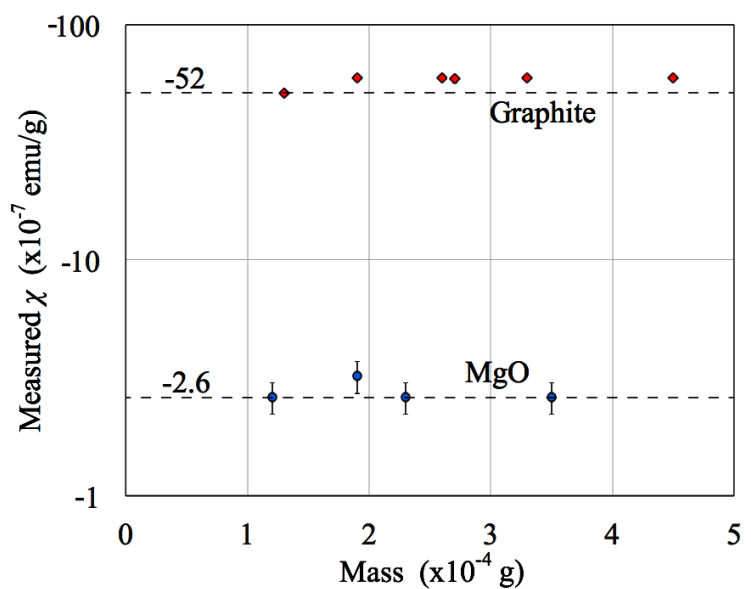

Fig. 2. Relationship between mass $m$ and $\chi_{\text {dia }}$ obtained for graphite particles. The $\chi_{\text {dia }}$ values were calculated from the translating velocity of the particles measured in the course of magnetic separation.

TABLE I

Numerical data of $\mathrm{MgO}$ and graphite particles used in the experiment of magnetic separation.

\begin{tabular}{c|c|c|c|c}
\hline \hline \multirow{2}{*}{ Sample } & mass & $v_{t}$ & $\chi_{\text {dia }}$ meas. & $\chi_{\text {dia }}$ other \\
\cline { 4 - 4 } & {$\left[\times 10^{-4} \mathrm{~g}\right]$} & {$[\mathrm{cm} / \mathrm{s}]$} & \multicolumn{2}{|c}{$\left[\times 10^{-7} \mathrm{emu} / \mathrm{g}\right]$} \\
\hline $\mathrm{MgO} \mathrm{\# 1}$ & 3.5 & 1.2 & $-2.6 \pm 0.4$ \\
$\mathrm{MgO} \mathrm{\# 2}$ & 1.2 & 1.2 & $-2.6 \pm 0.4$ \\
$\mathrm{MgO} \mathrm{\# 3}$ & 1.9 & 1.3 & $-3.2 \pm 0.5$ \\
$\mathrm{MgO} \mathrm{\# 4}$ & 2.3 & 1.2 & $-2.6 \pm 0.4$ \\
graphite \#1 & 2.6 & 5.4 & $-60 \pm 7$ \\
graphite \#2 & 2.7 & 5.4 & $-59 \pm 2$ \\
graphite \#3 & 3.3 & 11 & $-56 \pm 1$ \\
graphite \#4 & 1.9 & 12 & $-59 \pm 2$ \\
graphite \#5 & 4.5 & 11 & $-60 \pm 2$ \\
graphite \#6 & 1.3 & 10 & $-52 \pm 2$ & \\
& & &
\end{tabular}

gradient in the $z$-direction. The alteration was expected to induce the release of diamagnetic samples with small $\left|\chi_{\text {dia }}\right|$ values.

Trajectory of the sample translation was recorded by the high-speed camera, CASIO EX-F1 (spatial resolution: $0.004 \mathrm{~cm}$, time resolution: $0.033 \mathrm{fps}$ ), which was set outside the glass tube [1]. Image of the sample motion was taken from the direction of a $-y$-axis. The $v-x$ relations of the samples were obtained from time-sequential photographs to obtain terminal velocity $v_{t}$ of the sample in the $B \sim 0$ region.

\section{Results and discussions}

An example of magnetic separation observed in the mixture of graphite and magnesia particle is shown in Fig. 1 as a time sequence photograph. The particles were separated into two different groups of material as they translated towards the direction of decreasing field 
(i.e. $+x$-axis). It is seen that the above-mentioned procedures were effective to realize the translation and separation of the particles It is also confirmed from the figure that separation is completed within the short duration of microgravity.

The $\chi_{\text {dia }}$ values (per unit mass) of the particles are obtained by inserting the numerical values of $v_{t}$ and $B_{0}$ in the equation of a energy conservation described as

$$
1 / 2 m v_{T}^{2}+1 / 2 m \chi_{d i a} B_{0}^{2}=0
$$

where $m$ denote mass of the particles $[1,4]$. As shown in Table I, the $\chi_{\text {dia }}$ values of the translated particles obtained in this manner agreed fairly well with the published values; the deviations between the measured and published values were probably caused by contamination of some small particles attached to the sample. It is generally difficult to detect the magnetization of a weak magnetic material from a reduced size sample (e.g. diameter $<0.5 \mathrm{~mm})$. This is because the interference of background signals emitted from the sample holder is not negligible; $m$ measurement of the sample becomes difficult as well. It was suggested that the $\chi_{\text {dia }}$ measurement based on field-induced translation could avoid the abovementioned difficulties [3].

The results of $\chi_{\text {dia }}$ measurement performed in different sample sizes are compiled in Table 1 as well as in Fig. 2, and no significant correlation were seen between $\chi_{d i a}$ and $m$. The data show that the translation of the particles followed the conservation rule of Eq.(1) throughout the separation process. In order to apply the present method of separation \& identification in various practical purposes, it is important to confirm the efficiency of the above-mentioned conservation rule at various sample sizes. In this sense, it is necessary to extend the $\chi_{\text {dia }}$ v.s. $m$ measurement in the micron-size $\&$ cm-size particles.
It was suggested that the significance of magnetic separation could be comparable to that of the chromatography separation commonly used to extract the organic molecules, because extraction of new solid phases from heterogeneous grain ensemble would lead to important discoveries [1]. However, the separation of diamagnetic particles was formerly possible in materials that had $\left|\chi_{\text {dia }}\right|$ values above $5 \times 10^{-7} \mathrm{emu} / \mathrm{g}$, whereas published $\left|\chi_{\text {dia }}\right|$ data of many materials ranged between $10^{-7}$ and $5 \times 10^{-7} \mathrm{emu} / \mathrm{g}$ [4] (i.e. calcite: 3.55 , cellulose: 4.2 , corundum: 3.60 , lead: 1.1 , silver: 1.9 , siliconcarbide: $3.2 \times 10^{-7} \mathrm{emu} / \mathrm{g}$ ).

In this sense, further technical improvement is required to realize the separation of diamagnetic materials in general, and the procedures introduced in the present study to translate the $\mathrm{MgO}$ particles may provide a breakthrough to achieve the separation.

\section{References}

[1] K. Hisayoshi, C. Uyeda, K. Terada, Sci. Rep. 6, 38431 (2010).

[2] For example, E. Beaugnon, R. Tournier, Nature 349, 470 (1991).

[3] C. Uyeda, K. Hisayoshi, S. Kanou, J. Phys. Soc. Jpn. 79, 064709 (2010).

[4] R. Gupta, Diamagnetism, Landort Bornstein, New Series II, Springer Berlin 1983, p. 445. 